\title{
Adoção da Web 2.0 em bibliotecas de universidades públicas espanholas: perspectivas de interação do bibliotecário com as redes sociais - relato de pesquisa
}

David Vernon Vieira

Sofia Galvão Baptista

Aurora Cuevas Cerveró

\author{
Universidade Federal do Ceará - Campus Cariri \\ Professor Assistente Curso de Biblioteconomia \\ Doutorando em Ciência da Informação- \\ Universidade de Brasília
}

Universidade de Brasília - Faculdade de Ciência da Informação. Professor Associado - Doutorado em Ciência da Informação

Universidad Complutense de Madrid - Facultad de Ciencias de la Documentación. Professor Doutorado em Documentação

O surgimento da Web Social proporcionou um espaço virtual de participação dos usuários de bibliotecas. Assim, casos de adoção de redes sociais têm sido observados nas bibliotecas universitárias espanholas desde 2005, quando se publicaram os primeiros artigos científicos para acompanhar este movimento. Considerando o âmbito das bibliotecas de universidades públicas na Espanha, foi realizada uma pesquisa exploratória para conhecer a situação real do modelo de presença dessas bibliotecas nas redes sociais Facebook e Twitter. Este trabalho investiga um modelo de adoção das redes sociais, considerando aspectos que envolvem criação dos perfis das bibliotecas, publicação de conteúdo, interação com os usuários, criação de conteúdo pelo usuário, estabelecimento de normas de uso e estilo e medição e avaliação dos indicadores sociais para apoiar gestores de bibliotecas universitárias, neste tipo de projeto tecnológico. Apesar do levantamento realizado constatar a adoção das mídias sociais nas bibliotecas universitárias espanholas, observa-se que ainda há necessidade de se oferecer uma experiência para o usuário que propicie a sua participação, através de comentários e outras ações que promovam a interação com o bibliotecário. 
Palavras-chave: Biblioteca 2.0; Interação; Web social; Bibliotecas universitárias; Redes sociais.

\section{Adoption of Web 2.0 in Spanish Public Universities Libraries: perspectives of librarian interaction with the Social Networks - a research report}

The rise of the Social Web has provided a virtual space for patron participation thus, cases of adoption of social networks have been observed in the Spanish university libraries since 2005 when it published the first scientific papers to follow this movement. Considering the universe of public university libraries in Spain this paper was performed an exploratory research to know the real situation of the model of presence of these libraries in social networks Facebook and Twitter present in this space. This paper investigates a model considering adoption of social networking aspects that involve the creation of profiles for libraries, publishing content, interaction with users, user generated content, establishing rules of use and style, and measurement and evaluation of social indicators to support the management of university libraries in this type of technological project. Although the survey finds that adoption of social media in University Libraries in Spain shows that there is still a need to provide a user experience that is to afford their participation through comments and other actions that promote the interaction with the librarian.

Keywords: Library 2.0; Interaction; Social web; Academic libraries; Social networks

Recebido em 14.11.2012 Aceito em.21.01.2013

\section{Introdução}

Apesar da comunidade de bibliotecários, seja no âmbito espanhol, como no internacional, já ter algum contato promissor com experiências de Biblioteca 2.0 com relativo êxito, alguns autores mencionam aspectos críticos quanto à adoção dos softwares sociais, utilizados pelas bibliotecas no decorrer dos últimos anos. 
As funcionalidades presentes nas ferramentas da Web Social oferecem diversos recursos para ajudar as bibliotecas a interagir e se comunicar com os seus usuários.

Em princípio, Franganillo e Catalán Vega (2005) consideravam os blogs e as ferramentas de agregação de conteúdo como espaços para difundir a informação no âmbito educativo e das pesquisas nas bibliotecas.

Já destacava Serrano Cobos (2007), dentre as características da Web 2.0, elementos que permitiam aos usuários de biblioteca interagir com uma arquitetura de informação participativa e democrática, que empregava software livre e disponibilizado através de aplicações via Web.

Arroyo Vázquez e Merlo Vega (2007) indicavam algumas experiências práticas de ferramentas que poderiam ser utilizadas em bibliotecas, como, por exemplo: os blogs, a agregação de conteúdo (do inglês content syndication), os podcasts, os espaços wiki, as ferramentas de bookmark social, através do del.icio.us ${ }^{1}$, Connotea ${ }^{2}$ e CiteULike ${ }^{3}$, a etiquetação social por meio do tagging ou folksonomías e os sítios Web para o compartilhamento de imagens, fotos ou vídeos, como, por exemplo a do Flickr ${ }^{4}$ e o Youtube ${ }^{5}$, como também a utilização das redes sociais, como o Orkut $^{6}$ e o Myspace ${ }^{7}$, que, por acaso, não tiveram sua adoção em terras espanholas e acabaram sendo substituídas depois pelo Facebook ${ }^{8}$ e pela Tuenti ${ }^{\text {. }}$.

Ao ampliar o espaço de utilização dos blogs em bibliotecas universitárias, López Hernández e Penadés de la Cruz (2007) destacavam o desenvolvimento de um blog para a promoção da leitura, observando as coleções de literatura de uma biblioteca universitária, no qual indicava o trajeto desta em uma campanha que incluía aquisições, exposições e resenhas bibliográficas.

Entretanto, em razão da Jornada profissional organizada pela Biblioteca Nacional da Espanha e pela Revista Educación y Biblioteca ${ }^{10}$, González Fernández-Villavicencio (2007) fez uma retrospectiva da história da Biblioteca 2.0 na Espanha, que indicava como marco teórico da Biblioteca 2.0 o artigo de Serrano Cobos (2006), publicado online e, logo depois, no Anuário Think EPI.

Neste trabalho, González Fernández-Villavicencio (2007) já anunciava como evolução dos blogs a aplicação de microblogging Twitter, que, ainda, não estava consolidada no meio bibliotecário e que, sem dúvida, tinha um futuro promissor para as bibliotecas, pois permitia estar em contato com os usuários, seja por meio de computadores ou celulares, como, também, da incorporação de funcionalidades da Web 2.0 aos

\footnotetext{
${ }^{1}$ Disponível em: <http://delicious.com/>. Acesso em: 10 nov. 2012.

2 Disponível em: <http://www.connotea.org/>. Acesso em: 10 nov. 2012.

${ }^{3}$ Disponível em: <http://www.citeulike.org/>. Acesso em: 10 nov. 2012.

${ }^{4}$ Disponível em: <http://www.flickr.com/>. Acesso em: 10 nov. 2012.

${ }^{5}$ Disponível em: <http://www.youtube.com>. Acesso em: 10 nov. 2012.

${ }^{6}$ Disponível em: <http://www.orkut.com>. Acesso em: 10 nov. 2012

${ }^{7}$ Disponível em: <http://www.myspace.com>. Acesso em: 10 nov. 2012.

${ }^{8}$ Disponível em: <http://www.facebook.com>. Acesso em: 10 nov. 2012.

Disponível em: <http://www.tuenti.com>. Acesso em: 10 nov. 2012.

${ }^{10}$ Revista Educación y Biblioteca, año 19, n. 161, p. 75-84, 2007. ISSN 0214-7491.
} 
Catálogos OPAC, conhecidos como OPAC Social, destacados por Margaix Arnal (2007).

Paralelamente, se estabeleceria um novo modelo de biblioteca no espaço das bibliotecas universitárias espanholas, conforme destacava Orera-Orera (2007). Esta biblioteca híbrida, que atenderia aos usuários presentes na sociedade da informação por meio de um modelo de gestão baseado na informática e nas telecomunicações e, também, no empenho com a formação dos usuários destas bibliotecas, por meio de programas de Alfabetização Informacional (ALFIN), que culminaram com a criação do Centro de Recursos para a Aprendizagem e a Pesquisa (CRAI, do castelhano: "Centro de Recursos para el Aprendizaje y la Investigación") nas Universidades Espanholas.

A concepção de uma biblioteca universitária, voltada à formação de usuários, propiciou a criação de programas de formação em competências informacionais que utilizariam as ferramentas da Web 2.0 para esta finalidade, como indica González Fernández-Villavicencio (2008). A autora acrescenta que esta aprendizagem é muitas vezes considerada como autoaprendizagem, autorreflexiva, cada vez mais interativa e baseada na experimentação. Além disso, esta interatividade, de acordo com Martínez Usero (2007), pode ser utilizada para: apresentar a informação de um modo mais atrativo e excitante; adotar como ferramenta de marketing, visando atrair usuários e/ou visitantes para conhecerem a biblioteca também fisicamente, além do espaço virtual; apresentar tutoriais de aprendizagem online interativos que avaliem os estudantes; permitir ao usuário, que interaja com comunidades virtuais de interesse e, ainda, favorecer o contato e a consulta a usuários através de enquetes online, questionários, votações e propiciar-Ihes a criação de conteúdos.

E, assim, González Fernández-Villavicencio (2008) completa: a Web 2.0, seus princípios e suas ferramentas estão modificando competências e atitudes informacionais, ao proporcionar geração de conteúdos pelos usuários e a colaboração online. Desta forma, o uso e a modificação da informação e o compartilhamento são elementos necessários para elaborar modelos, níveis e práticas da ALFIN.

Contudo, o que se viu, na prática, ao longo da adoção destas ferramentas 2.0 pelas bibliotecas espanholas, foi: a baixa interatividade e o pouco interesse pela colaboração dos usuários de biblioteca; e a falta de uma "Atitude 2.0" pelos administradores de biblioteca. O que pode ser notado pelas palavras de Merlo Vega (2007), ao destacar esta mudança de atitude de uma biblioteca passiva, que emprega serviços e recursos tecnológicos sem reverter isso para os usuários, para uma biblioteca interativa, na qual as tecnologias são um meio de se relacionar efetivamente com os usuários.

Ainda, para reforçar esta "Atitude 2.0", Martín (2009) esclarece quais seriam as atitudes de um bibliotecário de sistemas e que seria desempenhado no seu fazer profissional: por uma atitude de serviço orientado ao usuário; mudança, desenvolvimento e crescimento profissional; curiosidade, criatividade que gere novos desafios; 
compromisso com os objetivos da organização; prospecção que antecipe tendências; dinâmica, proativa, ética, respeito pela organização e aprendizagem constante. Tais atitudes demonstram que o bibliotecário 2.0 não está lidando somente com uma questão temporária, mas está procurando experimentar, antecipar tendências de comunicação e interação com os usuários, procurando atender suas necessidades, no atual contexto tecnológico.

Desta forma, o êxito momentâneo das mídias sociais com a experiência social, oferecida aos usuários destas tecnologias, conforme indica Rodríguez Fernández (2011), deixará de ser passageiro, devendo se tornar algo habitual na interação do usuário com a biblioteca. Contudo, um bom plano de comunicação da biblioteca não é suficiente. O alinhamento das "iniciativas 2.0" com o planejamento estratégico, desenvolvido pela biblioteca, também deve ser contemplado. Conforme exemplo do planejamento estratégico da "Universitat de Barcelona" para sua biblioteca, percebe-se que as diretrizes do planejamento determinam a melhoria dos canais de comunicação e participação dos usuários, demonstrando, dessa maneira, a preocupação em priorizar o atendimento aos usuários, potencializando a adoção das mídias sociais. Cabe salientar, de acordo com o que pode ser observado no documento do planejamento estratégico para o exercício de 2011 a 2014, que inclui, em sua linha estratégica, a abrangência de "Sociedade e a Comunicação" aborda "a melhoria dos canais de comunicação e participação dos usuários" (vide página 30 do referido documento ${ }^{11}$ ).

Assim, a gestão do projeto de mídia social deverá disponibilizar um conteúdo adequado e legal, ou seja, capaz de atender aos requisitos de propriedade intelectual, com licenças de documentação do tipo Copyleft ou Creative Commons, como deixa claro Rodríguez Fernández (2011). Neste sentido, a elaboração de "Guias de Uso e Estilo das Redes Sociais" pelas bibliotecas, deverá ser direcionado no sentido de normatizar estes aspectos, conforme exemplos das Bibliotecas Públicas de la Generalitat de Catalunya $^{12}$ ou da Biblioteca da Universidade de Sevilla ${ }^{13}$.

Neste contexto, para operacionalizar tais atividades, o bibliotecário deverá ter experiência com as plataformas das redes sociais e, assim, poderia ser eleito para exercer a figura de Community Manager (Líder da Comunidade Virtual) da biblioteca. De acordo com Marquina (2010), esta figura seria o profissional encarregado não somente pelas redes sociais, mas, também, por qualquer atividade e função relacionada à Internet, à presença da biblioteca na rede e à geração de conteúdo online.

\footnotetext{
${ }^{11}$ Disponível em: <http://www.bib.ub.edu/fileadmin/arxius/plaestrategic2011-14.pdf>. Acesso em: 11 dez. 2012.

${ }_{12}$ Disponível em: <http://www.gencat.cat/xarxessocials/pdf/v1_guia_usos_xarxa_es.pdf>. Acesso em: 11 dez. 2012.

${ }_{13}$ Disponível em: <http://pt.scribd.com/doc/67194912/Guia-de-Uso-de-la-Web-Social-en-la-BUS-2011>. Acesso em: $11 \mathrm{dez} .2012$.
} 


\section{Objeto de estudo e metodologia}

$\mathrm{Na}$ Espanha, o processo de adoção das redes sociais pelas bibliotecas universitárias tem sido tema de discussão por diversos artigos científicos e bibliotecários em encontros profissionais. Muitos projetos têm como horizonte melhorar a comunicação com os usuários e, também, marcar a presença da biblioteca na Web Social. Assim sendo, depois de quase seis anos de surgimento destas ferramentas no espaço bibliotecário, a situação evidencia algumas características.

Esta pesquisa teve como proposta conhecer a situação atual da adoção de duas ferramentas sociais no espaço das bibliotecas universitárias espanholas e, também, formular um modelo de adoção das redes sociais nas bibliotecas universitárias. O modelo poderá servir para análise e reflexão dos responsáveis por novos projetos e, igualmente, para os gestores utilizarem como instrumento de apoio e, assim, acompanhar todo o processo.

Definiram-se os seguintes objetivos:

a) identificar a situação da adoção das redes sociais por bibliotecas universitárias espanholas: estabelecer as ferramentas sociais mais adotadas; e conhecer algumas iniciativas de interação com os usuários;

b) analisar um modelo de adoção das redes sociais para as bibliotecas universitárias: identificar iniciativas universitárias espanholas para cada uma das fases do modelo.

Para a realização de análise do modelo, baseou-se em dados obtidos de várias fontes de informação:

a)artigos de revistas e periódicos;

b) portais de bibliotecas universitárias espanholas;

d) documentação oferecida por algumas bibliotecas universitárias visitadas;

e) documentação obtida em congressos, encontros e seminários; e

f) conclusões das entrevistas realizadas com bibliotecários responsáveis pela atualização das redes sociais e diretores de bibliotecas universitárias.

\section{Resultados}

Para coletar os dados da pesquisa, selecionou-se 48 bibliotecas universitárias do tipo públicas, que estão disponíveis na lista da Rede de Bibliotecas Universitárias Espanholas (REBIUN) ${ }^{14}$. O levantamento foi

\footnotetext{
${ }^{14}$ Disponível em:<http://www.rebiun.org/bibliotecas.html>. Acesso em: 15 out. 2012.
} 
realizado em agosto de 2012 . Em seguida, procurou-se no sítio Web, da referida biblioteca, links que indicassem perfil nas plataformas de redes sociais Facebook e Twitter.

É importante destacar, dentro daquilo que foi observado no levantamento realizado, que foram encontrados distintos modelos de presença da biblioteca nas redes sociais. A presença do perfil da biblioteca nas redes sociais é feita:

a) por meio de um perfil próprio da universidade, aqui classificado como do tipo UNI;

b) um perfil próprio da biblioteca, comum a todos os campus ou perfil pertencente às faculdades, aqui classificado como tipo BIB;

c) um perfil diferenciado para cada biblioteca relacionada a uma faculdade ligada ao campus universitário, classificado como BIF.

Assim, a tabela, a seguir, representa os perfis relacionados às bibliotecas de universidades públicas espanholas, considerando as comunidades autônomas, conforme a lista: 1-AND Andaluzia; 2-ARA Aragón; 3-CAN Cantabria; 4-CLMCastilla La Mancha; 5-CLN Castilla y León; 6-CAT Catalunia; 7-CVA Comunitat Valenciana; 8-CMD Comunidad de Madrid; 9-EXT Extremadura; 10-GAL Galícia; 11-IBA Ilhas Baleares; 12-ICA Ilhas Canárias; 13-LRJ La Rioja; 14-NAV Foral de Navarra; 15-PAS Principado de Asturias; 16-PAV País Basco; 17-MUR Múrcia. 
Tabela 1 - Situação e Perfis de Redes Sociais das Bibliotecas de Universidades Públicas Espanholas

\begin{tabular}{|c|c|c|c|c|}
\hline \multirow[b]{2}{*}{$\mathrm{COM}$} & \multirow[b]{2}{*}{ Universidade Pública } & \multicolumn{2}{|l|}{ Tipo } & \multirow[b]{2}{*}{ Twitter } \\
\hline & & Perfil & Facebook & \\
\hline \multirow{9}{*}{1 AND } & Almería & UNI & "universidaddealmeria & @ualmeria \\
\hline & Cádiz & BIB & BibliotecaUCA & @Bibliouca \\
\hline & Córdoba & UNI & biblioteca.uco.es & @Univcordoba \\
\hline & Granada & UNI & universidadgranada & @CanalUGR \\
\hline & $\begin{array}{l}\text { Huelva } \\
\text { Internacional de } \\
\text { Andalucía }\end{array}$ & $\begin{array}{l}\text { BIB } \\
\text { UNI }\end{array}$ & $\begin{array}{c}\text { BibliotecaUniversidadHuelva } \\
\text { Universidad-Internacional-de- } \\
\text { Andalucía }\end{array}$ & $\begin{array}{l}\text { @bibliotecaUHU } \\
\text { @UNIAuniversidad }\end{array}$ \\
\hline & Jaén & BIB & $\begin{array}{l}\text { BUJA-Biblioteca-de-la- } \\
\text { Universidad-de-Jaén }\end{array}$ & @buja2009 \\
\hline & Málaga & $\mathrm{BIB}$ & BibliotecaUMA & BibliotecaUMA \\
\hline & Pablo de Olavide & $\mathrm{BIB}$ & bibupo & @bibupo \\
\hline & Sevilla & $\mathrm{BIB}$ & biblioteca.universidad.sevilla & @Biblioteca_US \\
\hline 2 ARA & Zaragoza & $\mathrm{BIB}$ & $\begin{array}{c}\text { Biblioteca.Universidad.de.Zara } \\
\text { goza }\end{array}$ & @bibliouz \\
\hline 3 CAN & Cantabria & $\mathrm{BIB}$ & $\begin{array}{c}\text { BibliotecadelaUniversidaddeCa } \\
\text { ntabria }\end{array}$ & @BUCantabria \\
\hline 4 CLM & Castilla La Mancha & BIB & bibliotecauniversitaria.uclm & $@ B U C L M$ \\
\hline \multirow{3}{*}{5 CLN } & Burgos & $\mathrm{BIB}$ & bibliotecaubu & @bibliotecaubu \\
\hline & $\begin{array}{l}\text { León } \\
\text { Salamanca }\end{array}$ & $\begin{array}{l}\text { UNI } \\
\text { BIB }\end{array}$ & $\begin{array}{c}\text { unileon } \\
\text { bibliousal }\end{array}$ & $\begin{array}{c}\text { @unileon } \\
\text { @bibliotecasusal }\end{array}$ \\
\hline & Valladolid & UNI & Universidad-de-Valladolid & @universidaddeva \\
\hline
\end{tabular}

Fonte: Dados da pesquisa. 
Tabela 1 - Situação e Perfis de Redes Sociais das Bibliotecas de Universidades Públicas Espanholas (continuação)

\begin{tabular}{|c|c|c|c|c|}
\hline COM & $\begin{array}{l}\text { Universidade } \\
\text { Pública }\end{array}$ & $\begin{array}{l}\text { Tipo } \\
\text { Perfil }\end{array}$ & Facebook & Twitter \\
\hline \multirow{7}{*}{6 CAT } & $\begin{array}{l}\text { Autónoma de } \\
\text { Barcelona }\end{array}$ & BIB & bchgUAB & @@bchgUAB \\
\hline & Barcelona & BIB & UBbiblio & @BibBiblio \\
\hline & Girona & $\mathrm{BIB}$ & BibliotecaUdG & @UdGBiblioteca \\
\hline & Lleida & UNI & universitatdelleida & @AM_UdL \\
\hline & $\begin{array}{l}\text { Politècnica de } \\
\text { Catalunya }\end{array}$ & BIB & bibliotequesUPC & @bibliotecnica \\
\hline & Pompeu Fabra & UNI & UniversitatPompeuFabra & @univpompeufabr \\
\hline & Rovira i Virgili & UNI & Non oficial Universitat-Rovira-i-Virgili & @urvtarragona \\
\hline \multirow{5}{*}{7 CVA } & Alicante & UNI & biblioteca.UA & @UA_Universidad \\
\hline & Jaume I & UNI & uji.val & @UJI_noticies \\
\hline & Miguel Hernandez & $\mathrm{BIB}$ & $\begin{array}{l}\text { biblioteca.universidadmiguelhernande } \\
\qquad z\end{array}$ & @UniversidadMH \\
\hline & $\begin{array}{l}\text { Politècnica de } \\
\text { València }\end{array}$ & BIB & BibliotequesUPV & @BiblioUPV \\
\hline & València & UNI & universitatdevalencia & @UV_EG \\
\hline \multirow{6}{*}{$8 \mathrm{CMD}$} & Alcalá & UNI & UniversidadDeAlcala & @UAHes \\
\hline & Autónoma de Madrid & $\mathrm{BIB}$ & BibliotecaArchivoUAM & @UAM_Madrid \\
\hline & Carlos III de Madrid & $\mathrm{BIB}$ & biblioteca.UC3M & @biblioteca_uc3m \\
\hline & $\begin{array}{l}\text { Complutense de } \\
\text { Madrid }\end{array}$ & $\mathrm{BIB}$ & BibliotecaComplutense & @BComplutense \\
\hline & $\begin{array}{l}\text { Politécnica de } \\
\text { Madrid }\end{array}$ & BIB & bibliotecaupm & @biblioupm \\
\hline & Rey Juan Carlos & BIB & biblioteca.urjc & $@ B U R J C$ \\
\hline 9 EXT & Extremadura & UNI & $\begin{array}{c}\text { People/biblioteca.universidaddeextrem } \\
\text { adura ou Universidad-de- } \\
\text { Extremadura-UEx }\end{array}$ & $\underset{n}{\text { @uexcomunicacio }}$ \\
\hline \multirow{3}{*}{$10 \mathrm{GAL}$} & Coruña & BIF & $\begin{array}{c}\text { Biblioteca-da-Universidade-da- } \\
\text { Coruña-Sección-de-Información-e- } \\
\text { Coordinación }\end{array}$ & @BibInformatica \\
\hline & $\begin{array}{l}\text { Santiago de } \\
\text { Compostela }\end{array}$ & BIB & $\begin{array}{c}\text { biblioteca.universitaria.santiago.comp } \\
\text { ostela }\end{array}$ & @BUSCUSC \\
\hline & Vigo & UNI & uvigo & @uvigo \\
\hline 11 IBA & Les Illes Barears & $\mathrm{BIB}$ & $\begin{array}{l}\text { Servei-de-Biblioteca-i-Documentació- } \\
\text { UIB }\end{array}$ & @BibliotecaUIB \\
\hline 12 ICA & $\begin{array}{l}\text { La Laguna } \\
\text { Las Palmas de Gran } \\
\text { Canaria }\end{array}$ & $\begin{array}{l}\text { BIB } \\
\text { BIB }\end{array}$ & $\begin{array}{l}\text { BibliotecaUniversidadLaLaguna } \\
\text { bulpgc }\end{array}$ & $\begin{array}{l}\text { @BibliotecaULL } \\
\text { @bulpgc }\end{array}$ \\
\hline 13 LRJ & La Rioja & $\mathrm{BIB}$ & Biblioteca-Universitaria-de-La-Rioja & @bibur \\
\hline $14 \mathrm{NAV}$ & Pública de Navarra & UNI & upnauniversidad & @UNavarra \\
\hline 15 PAS & Oviedo & $\mathrm{BIB}$ & Buniovi & $@$ @iraelBUO \\
\hline 16 PAV & País Vasco & UNI & pages/UPVEHU/106770286010768 & @upvehu \\
\hline \multirow[b]{2}{*}{17 MUR } & Murcia & $\mathrm{BIB}$ & biblioteca.universidaddemurcia & @bibliotecaumu \\
\hline & $\begin{array}{l}\text { Politécnica de } \\
\text { Cartagena }\end{array}$ & $\mathrm{BIB}$ & Servicio de Documentación UPCT & @BibliotecaUPCT \\
\hline
\end{tabular}

Fonte: Dados da pesquisa.

O modelo de adoção das redes sociais para bibliotecas universitárias baseia-se nos três componentes do modelo de Biblioteca Universitária 2.0, propostos por Xu, Ouyang e Chu (2009), o bibliotecário 2.0, o usuário 2.0 e a informação 2.0. Estes componentes são dinâmicos e interativos, fazendo com que o potencial de criar conteúdo pelo usuário, nas mídias sociais, esteja presente no Catálogo OPAC 2.0. 
À medida que o assunto postado pelo bibliotecário no perfil da rede social ou no microblog flui, ele passa a assumir a dinâmica da Informação 2.0, podendo gerar interesse dos usuários, os quais podem comentar, curtir ou, simplesmente, ler e manter-se engajados. Este engajamento irá depender, também, da possibilidade de cocriação da experiência online, que a biblioteca/bibliotecário irá oferecer ao usuário (GEROLIMOS, 2011). O modelo, a seguir, representa as fases de adoção das mídias sociais pela Biblioteca, observando alguns aspectos, como: criação do perfil, publicação de conteúdo, interação do usuário a partir do conteúdo publicado, criação de conteúdo pelo usuário, planejamento e estabelecimento de normas de uso e avaliação das métricas de uso, conforme a Figura 1.

Figura 1 - Modelo de adoção das redes sociais em bibliotecas universitárias

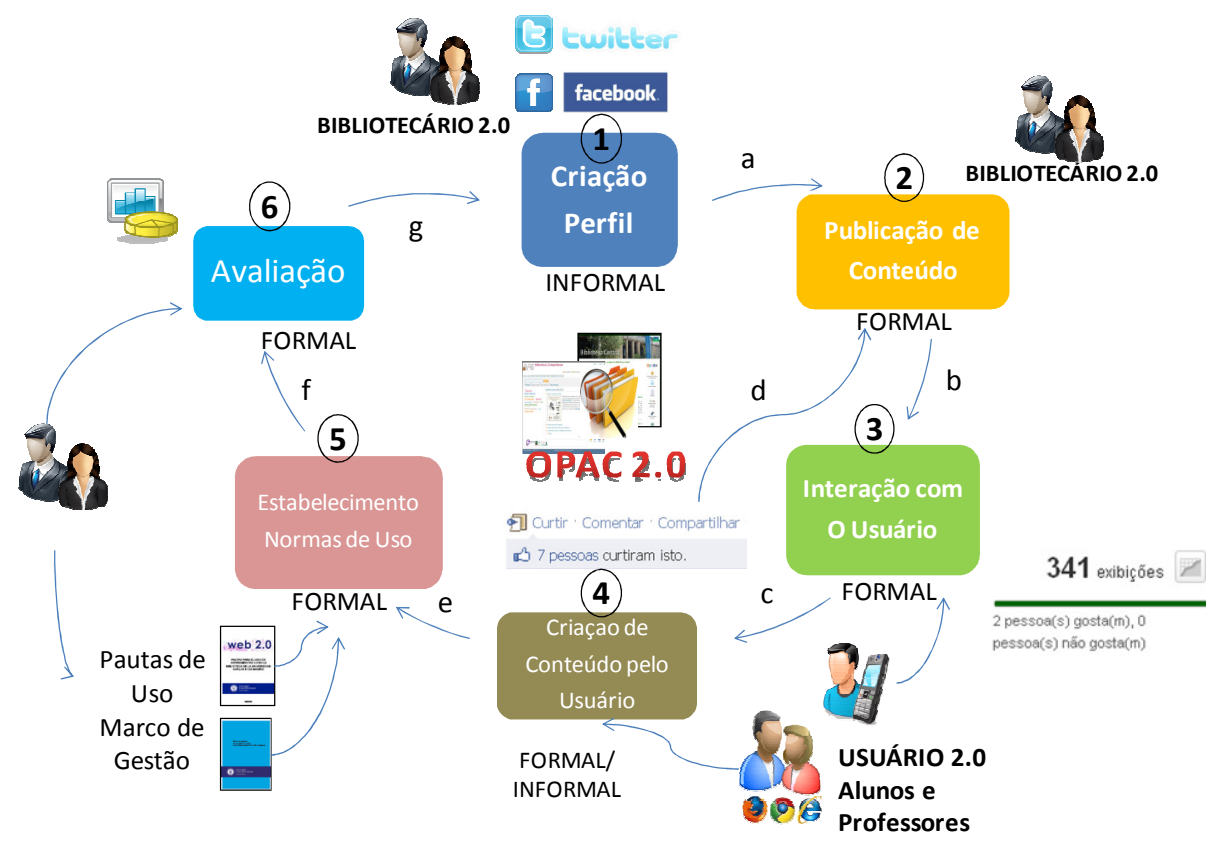

Fonte: Elaborado pelos autores.

A fase 1, de "Criação do Perfil", representa o início da adoção das mídias sociais na biblioteca. Neste momento, o bibliotecário escolhe a forma como a biblioteca irá se apresentar perante a rede social escolhida, conforme destacou Margaix-Arnal (2007), ou no microblog. Embora muitos bibliotecários tenham seu próprio perfil social, o perfil a ser criado irá representar a biblioteca perante os seus usuários, que podem ser internos ou externos à instituição. Assim, deverão ser observados aspectos humanos de etiqueta e comunicação da informação, além dos tecnológicos, envolvendo capacitação e equipamentos necessários para o seu uso, com vistas a tornar esta fase exitosa, conforme o documento "Pautas para Uso das ferramentas 2.0 na Biblioteca da Universidade 
Carlos III de Madri"15 ou o "Guia de Uso e Estilo das Redes Sociais" usados pelo Governo da Catalunia ${ }^{16}$, embora o Informe da Associação Profissional de Especialistas em Informação (APEI) ${ }^{17}$ sobre Web social, elaborado por Margaix-Arnal (2008), resuma várias questões relativas a bibliotecas. O aspecto da informalidade na sua adoção ainda está presente neste momento.

A fase 2, de "Publicação de Conteúdo", representa a edição de notícias, eventos e tutoriais, ou seja, todo material necessário para a biblioteca poder se comunicar com os usuários em potencial. Neste caso, a comunicação assume um perfil formal, no qual a biblioteca divulga algo oficial para seus usuários.

A fase 3, que é a de "Interação com o Usuário", representa iniciativas que o usuário adota para interagir com a biblioteca: ter cadastro no perfil da biblioteca para receber notícias; receber uma resposta a uma publicação feita pela biblioteca através de um microblog; um comentário a uma publicação feita pela biblioteca através de uma rede social ou blog; um retweet de uma publicação feita pela biblioteca, por meio de um microblog. Cabe destacar, aqui, que a convergência tecnológica permite aos usuários da biblioteca acessar estas plataformas para interagir, seja através de computador ou dispositivos móveis, como celulares e tablets. As letras " $b$ " e "d", indicadas na figura 1 , representam estes momentos.

A fase 4, de "Criação de Conteúdo pelo Usuário", representa iniciativas de algum comentário feito pelo usuário a uma publicação realizada pelo bibliotecário. O usuário pode, também, publicar conteúdo em "mural" ou "grupo de discussão" disponibilizado pela biblioteca em seu perfil na rede social, o que pode gerar interesse e repercussão para a biblioteca. O aspecto de comunicação pode ser informal por parte do usuário, que pode gerar uma resposta formal do bibliotecário, representando a biblioteca. As letras " $d$ " e " $e$ " irão representar este momento.

A fase 5, de "Estabelecimento de normas de uso", será determinada no momento em que a biblioteca/instituição ${ }^{18}$ necessitará instituir uma linha de ação e gestão das atividades pertinentes ao emprego das mídias sociais pela biblioteca. Para isso, a biblioteca deve formalizar algum documento, estabelecendo como será utilizada cada uma das mídias sociais perfiladas e a instituição a qual pertence a biblioteca definirá, documentalmente, as diretrizes a serem observadas para 0 acompanhamento de uso destas ferramentas. Nesta fase, também, será definido qual perfil será escolhido para o bibliotecário que irá interagir com estas mídias sociais, bem como o investimento ou capacitação necessários para este profissional lidar com as mídias selecionadas pela biblioteca. Por

15 Disponível em: <http://www.uc3m.es/portal/page/portal/biblioteca/sobre_la_biblioteca/pautas-para-usoherramientas-2-0.pdf >. Acesso em: 20 nov. 2012.

16 Disponível em: <http://www.gencat.cat/xarxessocials/pdf/v1_guia_usos_xarxa_es.pdf >. Acesso em: 22 nov. 2012.

${ }^{17}$ Disponível em: < http://hdl.handle.net/10760/12506>. Acesso em: 22 nov. 2012.

${ }^{18}$ A instituição, neste caso, é a Universidade que disponibiliza seu perfil nas redes sociais para a biblioteca se comunicar com os usuários. 


\section{último, que tipo de informação será disponibilizado nos perfis da biblioteca} nas mídias sociais.

A fase 6, de "Avaliação", representa o momento em que as atividades/ações realizadas nas mídias sociais serão medidas e analisadas pela direção, para incremento nas atividades ou cancelamento de perfil, caso o retorno não tenha sido positivo, através de indicadores estabelecidos para uso mídias sociais pela biblioteca.

Assim, a capacidade de criação de experiências online será o resultado da organização destas fases acima descritas, para desenvolver o aspecto da interação do bibliotecário com o usuário, por meio das mídias sociais presentes nas plataformas ligadas ao Catálogo OPAC da biblioteca universitária.

O Quadro 1 representa as fases do modelo de adoção, juntamente com a necessidade de interação e a situação expressa encontrada nas bibliotecas pesquisadas.

Quadro 1 - Modelo de adoção das Mídias Sociais na Biblioteca Universitária

\begin{tabular}{|c|c|c|c|}
\hline & Fase da Adoção & Necessidade de Interação & Situação Expressa \\
\hline 1 & Criação do Perfil & $\begin{array}{l}\text { - Observar a qual público irá se dirigir a mídia } \\
\text { social; } \\
\text { - Analisar qual (is) é (são) o(s) potencial (is) de } \\
\text { interação da mídia social escolhida. }\end{array}$ & $\begin{array}{l}\text { - A rede social Tuenti possui um público } \\
\text { diferente da rede social Facebook. } \\
\text { - A rede social Facebook permite "Curtir" } \\
\text { uma notícia, comentar e compartilhar. }\end{array}$ \\
\hline 2 & $\begin{array}{l}\text { Publicação de } \\
\text { Conteúdo }\end{array}$ & $\begin{array}{l}\text { - O conteúdo deve ser publicado de forma diária } \\
\text { para manter os usuários mais próximos. } \\
\text { - O conteúdo deve ser de acordo com o assunto } \\
\text { abordado pelos cursos vinculados àquela } \\
\text { Biblioteca. }\end{array}$ & $\begin{array}{l}\text { - A Biblioteca ligada a uma Faculdade } \\
\text { deve publicar notícias especializadas na } \\
\text { área que atua. } \\
\text { - As Bibliotecas devem possuir em cada } \\
\text { Campus, ao menos um bibliotecário } 2.0 \\
\text { que irá atender ao usuário e dar suporte } \\
\text { ao pesquisador. }\end{array}$ \\
\hline 3 & $\begin{array}{l}\text { Interação como } \\
\text { Usuário }\end{array}$ & $\begin{array}{l}\text { - O acompanhamento da interação deve ser } \\
\text { contínuo, ensejando novas experiências e induzir } \\
\text { o usuário a interagir baseado nas necessidades } \\
\text { de informação por ele pretendida. }\end{array}$ & $\begin{array}{l}\text { - A necessidade de um estudo de um } \\
\text { usuário faz com que o assunto abordado } \\
\text { provoque uma resposta do usuário ao } \\
\text { assunto publicado. } \\
\text { - Reuniões para promover os serviços da } \\
\text { biblioteca junto aos representantes devem } \\
\text { tratar das necessidades. }\end{array}$ \\
\hline 4 & $\begin{array}{l}\text { Criação de } \\
\text { Conteúdo pelo } \\
\text { Usuário }\end{array}$ & $\begin{array}{l}\text { - Se o catálogo OPAC pesquisado não trouxer } \\
\text { funcionalidades presentes no Catálogo Social } \\
\text { OPAC. É preciso fazer a mudança para esta nova } \\
\text { versão para obter maior interação. } \\
\text { - Iniciativas de interação devem ser } \\
\text { disponibilizadas para provocar a criação de } \\
\text { conteúdo pelo usuário. }\end{array}$ & $\begin{array}{l}\text { - Iniciativas de Interação em blogs: Clube } \\
\text { da Leitura; Concursos de Fotografia; } \\
\text { Sugestões de livros, CDs, DVDs para } \\
\text { atender a um público ou data específica } \\
\text { ajudam a produzir resenhas e a } \\
\text { participação dos usuários. } \\
\text { - Iniciativas de interação nas redes sociais: } \\
\text { Enquetes para saber necessidades dos } \\
\text { usuários. } \\
\text { - Grupos de discussão; Temas mais } \\
\text { relevantes; Assuntos mais lidos e mais } \\
\text { comentados. }\end{array}$ \\
\hline 5 & $\begin{array}{l}\text { Estabelecimento de } \\
\text { normas de uso }\end{array}$ & $\begin{array}{l}\text { - Documento que indica de que forma as mídias } \\
\text { sociais serão utilizadas ajudam no } \\
\text { acompanhamento. } \\
\text { - Documento para gerenciar as mídias sociais } \\
\text { adotadas estabelece indicadores. }\end{array}$ & $\begin{array}{l}\text { - Documento de Pautas de Uso } \\
\text { - Documento de Marco de Gestão com } \\
\text { indicadores }\end{array}$ \\
\hline 6 & $\begin{array}{l}\text { Avaliação das } \\
\text { mídias sociais }\end{array}$ & $\begin{array}{l}\text { - Baseado em indicadores estabelecidos na fase } \\
\text { anterior a Direção da Biblioteca Universitária } \\
\text { organiza as iniciativas e define diretrizes. }\end{array}$ & $\begin{array}{l}\text { - Selecionar softwares/ aplicativos via web } \\
\text { para acompanhar os indicadores. } \\
\text { - Acompanhar e apresentar os indicadores } \\
\text { para o relatório anual. }\end{array}$ \\
\hline
\end{tabular}

Fonte: Elaborado pelos autores. 


\section{Conclusões}

Apesar do levantamento realizado constatar que todas as bibliotecas de universidades públicas espanholas adotaram alguma das plataformas usadas como ferramenta 2.0, não podemos afirmar que isso indique o sucesso neste processo de adoção.

A interação humana é o elemento principal neste contexto social tecnológico, porque não se trata simplesmente de usar este tipo de tecnologia como meio de comunicação com os seus usuários, conforme afirmaram os entrevistados. Nem, tão pouco, formalizar todas as fases do processo de adoção, mas proporcionar uma experiência para o usuário de bibliotecas universitárias capaz de creditar estas ferramentas como algo de que o usuário venha a participar.

Para Bradley e McDonald (2012), o resultado final seria aproveitar a colaboração em massa, proporcionada pela "Inteligência Coletiva". Porém, o que se pode observar é que isso ainda não está incorporado na missão da biblioteca, quando o assunto é a adoção deste tipo de ferramenta, para melhorar a relação com seus usuários.

Dentre as fases do modelo de adoção das mídias sociais destacados acima, aquelas que foram mais observadas foram as fases de criação de perfil e publicação de conteúdo como as mais utilizadas atualmente. Porém, percebe-se que as fases de estabelecimento de normas de uso e avaliação do uso das mídias sociais são a maior preocupação para a continuidade desta atividade, neste momento em que as bibliotecas passam por recortes nos investimentos provocados pela crise espanhola.

Assim, verifica-se que o próximo passo a ser dado estará relacionado com iniciativas que liderem os projetos de redes sociais nas bibliotecas universitárias, no sentido de incorporar indicadores de gestão, para saber onde e quando é apropriada a colaboração da comunidade de usuários.

\section{Agradecimento}

Os autores agradecem a Coordenação de Apoio à Pesquisa, pela concessão de uma bolsa de estudos para a realização desta pesquisa.

\section{Referências}

ARROYO VÁZQUEZ, N; MERLO VEGA, J. A. La biblioteca como usuaria de la web 2.0. In: JORNADAS ESPAÑOLAS DE DOCUMENTACIÓN, 10., 2007, Santiago de Compostela. Anais... Santiago de Compostela: FESABID, 2007. p. 1-11.

BRADLEY, A. J.; McDONALD, M. P. La organización social: convertir en resultados las oportunidades de las redes sociales. Barcelona: Profit Editorial, 2012.

FRANGANILLO, J; CATALÁN VEGA, M. A. Bitácoras y sindicación de contenidos: dos herramientas para difundir información. BiD: textos universitaris de biblioteconomia i documentació, n. 15, dez. 2005. 
Disponível em: <http://www.ub.edu/bid/15frang2.htm>. Acesso em: 25 set. 2012.

GEROLIMOS, Michalis. Academic Libraries on Facebook: An Analysis of Users' Comments. D-Lib Magazine, v. 17, n. 11/12, 2011. Disponível em: $<$

http://www.dlib.org/dlib/november11/gerolimos/11gerolimos.print.html> . Acesso em: 04 abr. 2012.

GONZÁLEZ FERNÁNDEZ-VILLAVICENCIO, N. Biblioteca 2.0 en España: el camino recorrido. Boletin de la Asociacion Andaluza de Bibliotecarios, v. 22, n. 86-87, p. 29-46, 2007.

GONZÁLEZ FERNÁNDEZ-VILLAVICENCIO, N. ALFIN 2.0: Herramientas 2.0 en programas ALFIN en bibliotecas de la Universidad de Sevilla. Boletín de la Asociación Andaluza de Bibliotecarios, v. 23, n. 92-93, p. 11-32, 2008.

LÓPEZ HERNÁNDEZ, F; PENADÉS DE LA CRUZ, H. 365 días de libros: blog para la promoción de la lectura. El profesional de la información, v. 16, n. 2, p. 131-133, mar./abr. 2007. Disponível em: <http://www.elprofesionaldelainformacion.com/contenidos/2007/marzo/0 6.pdf $>$. Acesso em: 25 set. 2012.

MARGAIX-ARNAL, D. El OPAC social, el catálogo en la Biblioteca 2.0: aplicación y posibilidades en las bibliotecas universitárias. In: JORNADAS ESPAÑOLAS DE DOCUMENTACIÓN, 10., 2007, Santiago de Compostela. Anais... Santiago de Compostela: FESABID, 2007. p. 199-205. Disponível em: <http://eprints.rclis.org/archive/00011103/>. Acesso em: 25 set. 2012.

MARGAIX-ARNAL, D. Las bibliotecas universitarias y Facebook: cómo y por qué estar presentes. El profesional de la información, v. 17, n. 6, p. 589601, nov./dez. 2008. Disponível em: <DOI: 10.3145/epi.2008.nov.02>. Acesso em: 28 set. 2012.

MARQUINA, J. La figura de community manager en las bibliotecas. Julian Marquina.es, 15 nov. 2010.2 Disponível em: <http://www.julianmarquina.es/figura-community-manager-bibliotecas>. Acesso em: 25 set. 2012.

MARTÍN, S. G. Bibliotecario de sistemas: una especialización con futuro. Información, Cultura y Sociedad, n. 21, p. 69-84, 2009. Disponível em: <http://www.scielo.org.ar/scielo.php?script=sci_arttext\&pid=S1851$17402009000200005 \& \mathrm{lng}=$ es\&nrm=iso>. Acesso em: 25 set. 2012.

MARTÍNEZ USERO, J. A. Nuevas tecnologías para nuevas bibliotecas: desarrollo de servicios de información electrónica. Buenos Aires: Alfagrama, 2007.

MERLO VEGA, J. A. Las tecnologías de la participación en las bibliotecas. Educación y Biblioteca, n. 161, p. 63-68, 2007.

ORERA-ORERA, L. La biblioteca universitaria ante el nuevo modelo social y educativo. El profesional de la información, v. 16, n. 4, p. 329-337, 
jul./ago.

2007.

Disponível

em: <http://www.elprofesionaldelainformacion.com/contenidos/2007/julio/06. pdf>. Acesso em: 25 set. 2012.

RODRÍGUEZ FERNÁNDEZ, Ó. Community manager. conviértete en experto en "social media". Madrid-ESP: Anaya Multimedia, 2011.

SERRANO COBOS, J. Web 2.0. Anuario ThinkEPI. 2007. p. 170-171. Disponível em: <http://www.thinkepi.net/web-20-en-las-bibliotecas-elconcepto-library-20>. Acesso em: 25 set. 2012.

SERRANO COBOS, J. Pasado, presente y futuro de la Web 2.0 en servicios de información digital. BiD: Textos Universitaris de Biblioteconomia $i$ Documentació, n. 17, $2006 . \quad$ Disponível em: <http://www.ub.edu/bid/17serra2.htm>. Acesso em: 25 set. 2012.

$\mathrm{XU}, \mathrm{C}$; O OUYANG, F.; CHU, H. The academic library meets web 2.0: applications and implications. The Journal of Academic Librarianship, v. 35, n. 4, p. 324-331, 2009. Disponível em: <http://dx.doi.org/10.1016/j.acalib.2009.04.003>. Acesso em: 25 set. 2012. 